\section{QUALITY OF LIFE IN PATIENTS WHO UNDERWENT PATENT FORAMEN OVALE CLOSURE, LEFT ATRIAL APPENDAGE CLOSURE OR MITRACLIP PROCEDURE}

${ }^{1}$ Tamara Naneishvili*, ${ }^{2}$ Lucy Cosbey, ${ }^{3}$ Chantal Patel, ${ }^{1}$ Adrian Large, ${ }^{1}$ Rob Butler. ${ }^{1}$ UHNM NHS Trust; ${ }^{2}$ UHNS NHS Trust; ${ }^{3}$ Keele University Medical School

\subsection{6/heartjn|-2017-311726.30}

Aim of study To assess patients quality of life (QOL) pre and post patent foramen ovale (PFO) closure, left atrial appendage (LAA) occlusion or Mitraclip procedures, in a single centre.

Material and Methods We analysed 148 patients who underwent one of the above procedures from October 2014 to October 2016 at RSUH. The mean age of patients who underwent PFO closure was 46 years, whilst in the LAA closure and Mitraclip procedure groups, the mean age was 78 and 82 years respectively. Data was entered into the NICOR database as part of the Commissioning through Evaluation process. We collected data using the EQ-5D-5L questionnaire, either in person or over the phone.

Results 67 patients underwent PFO closure, out of which 32 responded pre op and 22 post op, despite multiple repeated attempts. 30 patients out of 54 who underwent LAA closure responded pre-procedure and 27 post procedure. 19 and 18 patients out of 27 who underwent Mitraclip procedures responded pre op and post op respectively; 6 deceased (MitraClips only).

There was no improvement of QOL post PFO closure in terms of walking, dressing, conducting ADL's, pain and global health score. However, we demonstrated an improvement in the level of anxiety and depression.

In the LAA closure group of patients there was also an improvement in anxiety and depression, but similarly no improvement was seen in the other categories.

There was a significant improvement globally in the QOL in patients who had undergone the Mitraclip procedure. Additionally, their global health score showed almost a two-fold increase.

Conclusion Patients in the PFO and LAA closure groups showed no improvement in QOL, other than in anxiety and depression. This result was expected because both procedures are performed to prevent a further thromboembolic event (PFO and LAA) or further bleed on an anticoagulant (LAA). We were surprised that anxiety and depression were improved, but further questioning revealed patients had high levels of worry that a further event could occur without treatment, and that treatment mitigated the risk.

There was a dramatic improvement in QOL after Mitraclip procedure, as expected in a disease modifying procedure. The mortality was due to pump failure (2) and non-cardiac causes (4).

\begin{tabular}{|c|c|c|c|c|c|c|c|}
\hline & \multicolumn{2}{|c|}{$\begin{array}{l}\text { PFO closure } \\
\text { (\%) }\end{array}$} & \multicolumn{2}{|c|}{$\begin{array}{l}\text { LAA closure } \\
\text { (\%) }\end{array}$} & \multicolumn{2}{|c|}{$\begin{array}{c}\text { MitraClip procedure } \\
\text { (\%) }\end{array}$} \\
\hline & & Pre op & Post op & Pre op & Post op & Pre op & Post op \\
\hline \multirow{4}{*}{$\begin{array}{l}\text { Problems } \\
\text { with } \\
\text { walking }\end{array}$} & None & 84 & 87 & 40 & 41 & 11 & 26 \\
\hline & Slight & 12 & 8 & 23 & 26 & 5 & 27 \\
\hline & Moderate & 3 & 4 & 30 & 26 & 32 & 33 \\
\hline & Severe & 0 & 0 & 7 & 7 & 52 & 11 \\
\hline \multirow{5}{*}{$\begin{array}{l}\text { Problems } \\
\text { with } \\
\text { dressing }\end{array}$} & None & 93 & 95 & 67 & 63 & 47 & 72 \\
\hline & Slight & 6 & 4 & 23 & 26 & 21 & 16 \\
\hline & Moderate & 0 & 0 & 7 & 0 & 26 & 11 \\
\hline & Severe & 0 & 0 & 3 & 0 & 0 & 0 \\
\hline & Unable & 0 & 0 & 0 & 10 & 4 & 0 \\
\hline \multirow{5}{*}{$\begin{array}{l}\text { Problems } \\
\text { with ADL's }\end{array}$} & None & 71 & 83 & 50 & 44 & 11 & 6 \\
\hline & Slight & 22 & 8 & 27 & 22 & 21 & 39 \\
\hline & Moderate & 6 & 4 & 13 & 18 & 16 & 28 \\
\hline & Severe & 0 & 4 & 3 & 7 & 37 & 16 \\
\hline & Unable & 0 & 0 & 7 & 7 & 16 & 0 \\
\hline \multirow[t]{4}{*}{ Pain } & None & 81 & 79 & 67 & 66 & 58 & 83 \\
\hline & Slight & 12 & 12 & 20 & 22 & 16 & 11 \\
\hline & Moderate & 6 & 8 & 13 & 11 & 21 & 6 \\
\hline & Severe & 0 & 0 & 0 & 0 & 5 & 0 \\
\hline \multirow{5}{*}{$\begin{array}{l}\text { Anxiety } \\
\text { and } \\
\text { depression }\end{array}$} & None & 56 & 79 & 70 & 81 & 68 & 88 \\
\hline & \begin{tabular}{|l|} 
Slight \\
\end{tabular} & 25 & 12 & 23 & 15 & 21 & 11 \\
\hline & Moderate & 9 & 4 & 7 & 4 & 0 & 0 \\
\hline & Severe & 9 & 4 & 0 & 0 & 10 & 0 \\
\hline & Extreme & 6 & 0 & 0 & 0 & 0 & 0 \\
\hline \multicolumn{2}{|c|}{$\begin{array}{l}\text { Global health score } \\
\text { Median (IQR) }\end{array}$} & $\begin{array}{c}80 \\
(67.5-90)\end{array}$ & $\begin{array}{c}90 \\
(75-90)\end{array}$ & $\begin{array}{c}67.5 \\
(50-80)\end{array}$ & $\begin{array}{c}70 \\
(50-80)\end{array}$ & $\begin{array}{c}35 \\
(20-50)\end{array}$ & $\begin{array}{c}57.5 \\
(45-76)\end{array}$ \\
\hline & & \multicolumn{2}{|c|}{ PFO closure } & \multicolumn{2}{|c|}{ LAA closure } & \multicolumn{2}{|c|}{ Mitraclip } \\
\hline \multirow{2}{*}{$\begin{array}{l}\text { Gender } \\
\text { (\%) }\end{array}$} & Male & \multicolumn{2}{|c|}{52} & \multicolumn{2}{|c|}{68} & \multicolumn{2}{|l|}{48} \\
\hline & Female & \multirow{2}{*}{\multicolumn{2}{|c|}{48}} & \multicolumn{2}{|l|}{32} & \multicolumn{2}{|l|}{52} \\
\hline \multirow{2}{*}{$\begin{array}{l}\text { Age } \\
\text { (years) }\end{array}$} & Median & \multirow{2}{*}{\multicolumn{2}{|c|}{\begin{tabular}{|l|}
46 \\
$39-51$
\end{tabular}}} & \multirow{2}{*}{\multicolumn{2}{|c|}{$\begin{array}{l}78 \\
72-82\end{array}$}} & \multirow{2}{*}{\multicolumn{2}{|c|}{$\begin{array}{l}82 \\
76-86\end{array}$}} \\
\hline & $\begin{array}{l}\text { Interquartile } \\
\text { range }\end{array}$ & & & & & & \\
\hline
\end{tabular}

